\title{
Production ligneuse des taillis d'eucalyptus : effet de la fréquence de coupe et de la densité de plantation
}

\author{
A. RIEDACKER **, C. KNOCKAERT *, A. ZAIDI * \\ * Division de Recherches et d'Expérimentations forestières \\ B.P. 763, Rabat-Agdal, Maroc \\ *: Station de Sylviculture et de Production \\ Centre de Recherches forestières de Nancy, Champenoux, F 54280 Seichamps
}

\begin{abstract}
Résumé
La production ligneuse aérienne des taillis d'Eucalyptus camaldulensis au Maroc dépend de la fréquence des coupes et de la densité de plantation.

Dans un carré latin, à 12 ans, pour un écartement de $3,5 \times 3,5 \mathrm{~m}$, lorsque le taillis est exploité tous Ies $4,6,8$ ou 10 ans, la production totale n'est pas significativement différente de celle des témoins, c'est-à-dire du traitement en futaie. Mais, lorsque l'exploitation est effectuéc tous les 2 ans, la production chute de 34 p. 100.

Dans un autre essai, à densité de plantation allant de 1100 plants/ha à 40000 plants/ha, la production totale des futaies à 10 ans augmente significativement lorsque la densité augmente de 1100 plants/ha jusqu'à 10000 plants $/$ ha ; elle passe de $40 \mathrm{~m}^{3}$ à $100 \mathrm{~m} /$ ha. Au-delà de cette densité, l'accroissement de production n'est plus significatif. Comme dans l'essai précédent, le traitement en taillis ne diminue la production totale que pour des rotations inféricures à 4 ans et cela quclle que soit la densité. Après 5 rotations de 2 ans, la production totale n'atteint que 25 à 40 p. 100 des peuplements témoins.
\end{abstract}

L'étude de l'évolution de la masse foliaire et de la masse des fruits permet partiellement d'expliquer les résultats obtenus. L'origine de la baisse de production des taillis classiques après trois rotations de plus de 10 ans est réexaminée à la lumière de ces résultats.

\section{Introduction}

Le taillis est l'un des plus vieux traitements sylvicoles. On connaît cependant mal les lois de croissance des peuplements ainsi traités. Aussi, avons-nous cherché à préciser l'influence d'une part de la densité de plantation et d'autre part de la fréquence de coupe sur la productivité des taillis d'Eucalyptus camaldulensis au Maroc. 


\subsection{Iffet de la densité de plantation}

Des études antérieures (RIEDACKER, 1973) ont montré que l'augmentation de la densité se traduisait par une augmentation de la production ligneuse aérienne totale des peuplements d'Eucalyptus camaldulensis traités en taillis à des rotations supérieures à 10 ans. (La rotation est définic ici comme étant lintervalle entre 2 coupes rases). En Mamora orientale, en zone relativement aride à moins de $400 \mathrm{~mm}$ de précipitations par an, la production ligneuse aérienne totale depuis la plantation de futaies de 17 ans atteignait $82 \mathrm{~m}^{3}, 95 \mathrm{~m}^{3}, 112 \mathrm{~m}^{3}, 122 \mathrm{~m}^{3}$ et $157 \mathrm{~m}^{3}$ pour des écartements respectivement de $6 \times 6 \mathrm{~m}, 4 \times 4 \mathrm{~m}, 3 \times 3 \mathrm{~m}, 2 \times 2 \mathrm{~m}$ et $1 \times 1 \mathrm{~m}$. Dans la présente étude, nous avons cherché à déterminer si des densités encore plus élevées allaient se traduire par des accroissements encore plus forts. D'après les résultats de Strinbeck \& Brown (1976), il semble qu'au-delà d'une certaine densité de plantation des platanes, l'aecroissement ligneux aérien n'augmente plus avec la densité, tout au moins en labsence d'exploitation. A 4 ans, la plantation à $4 \times 4$ pieds $(1,2 \times 1,2 \mathrm{~m})$ fournit 46,8 tonnes de matière ligneuse aérienne fraîche par acre contre 44,2 pour un ścartement de $1 \times 4$ pieds $(0,3 \mathrm{~m} \times 1,2 \mathrm{~m})$.

\subsection{Effei de la fróquence de coupe}

L'exploitation annuelle ou bisannuelle des peuplements de platanes plantés à $1 \times 4$ picds se traduit par une baisse de production totale respectivement de 39 p. 100 et 12 p. 100 par mapport au témoin non coupé. Lorsque l'écartement est plus élevé $(4 \times 4$ pieds), l'exploitation annuelle ou bisannuelle se traduit par des chutes de production plas imporantes, respectivement de 49 p. 100 ei 39 p. 100.

Pour des densités trés élevées, il est possible qu'il soit nécessaire de couper plus fréquemment les peuplements pour éviter l’installation d'une concurrence aérienne trop forte et trop prolongéc susceptible de se traduire par un mortalité ou un alfaiblissenent important de tous les individus ou des individus qui auront été les plus dominés.

Lorsque les exploitations sont moins fréquentes, il arrive également que l'on observe des baisses de production. C'est ainsi qu'au Maroc, nous avons constaté dans des peuplements d'Eucalyplus camaldalensis exploités à des rotations de 12 à 14 ans (c'est-à-dire après l'âge auquel cst atteint le maximum d'accroissement ligneux moyen annucl) des baisses d'accroissements sensibles après la troisième rotation (RIEDACKER, 1973). Plusieurs hypothèses avaient alors été émises pour expliquer ce phénomène ; cela pouvait être dî au vicillissement normal du système racinaire, à la répétition des coupes plus ou moins bien supportées par les arbres, ou encore à l'épuisement minéral du sol. Los expériences décrites ci-dessous ont également été réalisées pour tente: d’élucider ce phénomène.

\section{Matériel et méthode}

En Mamora Centrale, à proximité de Rabat, sur sol sableux sous climat aride (450 mm de pluie par an), nous avons installé deux dispositifs expérimentaux (RIEDACKER, 1973). 


\subsection{Expérimentation $n^{\prime \prime} 1$}

Dans une plantation d'Eucalyptus camaldulensis plantéc à $3.5 \times 3.5 \mathrm{~m}$ au printcmps 1968 sur un sol riche (sables rouges), (ArTicues \& LEPOUTRE, 1968), nous avons installé au printemps 1970, sur une superficie clôturée de 2.82 há. un carré latin comprenant 6 traitements :

- le témoin non exploité (T);

- des traitements avec une coupe à blanc, début avril, tous les 2 ans (T 2), 4 ans (T 4), 6 ans (T 6), 8 ans (T 8), et tous les 10 ans (T 10).

Chaque carré élémentaire du carré latin comprenait 64 plants. En boldure, nous avions installé une répétition supplémentaire de chaque traitement pour établir des tarifs en volume ou en poids sec à l'étuve, en particulier pour le témoin (T) et pour calculs dans les intervalles entre les coupes à blanc pour les autres traitements. Lá méthode d'échantillonnage a été mise au point antéricurement (Bouciron et RibDAckER, 1973).

Pour chaque traitement, nous avons échantillonné 25 à 30 tiges, mesuré leur volume total ligneux aérien et le poids frais de leurs feuilles. Des échantillons de feuilles fraîches, d'environ $200 \mathrm{~g}$, ont été pesés frais, puis séchés à l'étuve jusqu'à poids constant, afin de déterminer le rapport poids frais-poids sec. Des équations de régression permettant d'obtenir le volume total de la tige ou le poids sec des feuilles à partir des circonférences des brins à $1,30 \mathrm{~m}$ ont ensuite été établies. Mais, dans les cépées de 2 ans, il y a en plus une multitude de brins feuillés de moins de $1 \mathrm{~m}$ de hauteur qui disparaissent ensuite. Leur masse foliaire a été déterminée dans une vingtaine de cépées de toutes dimensions, afin de déterminer le poids foliaire moyen pour les petits rejets. Notons enfin que $1 \mathrm{~kg}$ de feuilles sèches représente environ $6,4 \mathrm{~m}^{2}$ de surface foliaire (une face).

\subsection{Expérimentation $n^{\prime \prime} 2$}

Au voisinage du dispositif expérimental précédent, mais sur un sol sableux plus pauvre en éléments nutritifs (sables beiges - classement Artigues \& Lepoutre, 1968), avaient été installés deux carrés latins d'une superficie de deux hectares environ chacun, et entourés de bandes de protection. Ils comprenaient 6 densités de plantations différentes, soit 36 carrés élémentaires (écartement : $0,5 \times 0,5 \mathrm{~m} ; 0,75 \times 0,75 \mathrm{~m}$; $1 \mathrm{~m} \times 1 \mathrm{~m} ; 1,50 \times 1,50 \mathrm{~m} ; 1,50 \times 3 \mathrm{~m} ; 3 \times 3 \mathrm{~m})$, soit respectivement 40000 plants à l'hectare ; $17770 ; 10000 ; 4440 ; 2220$ et 1111 plants à l'hectare $\left(D_{40}, D_{17}, D_{16,}\right.$, $\left.\mathrm{D}_{1}, \mathrm{D}_{2}, \mathrm{D}_{1}\right)$.

Faute de pouvoir installer un dispositif expérimental supplémentaire, celui-ci a été remanié pour permettre l'étude simultanée de l'effet de la densité et de la fréquence des coupes; cxploitation tous les 2 ans $(0,35$ ha); tous les 3 ans $(1,04$ ha) ; tous les 4 ans $(0,86$ ha) et non exploités $(0,69$ ha). Pour des raisons pratiques, ces traitements sans répétition ont été superposés aux carrés latins. Une interprétation statistique en est donc exclue. Cependant, étant donné qu'il n'existe actuellement pas d'expériences de ce genre, nous avons jugé utile de présenter les résultats obtenus, car ils fournissent des indications précieuses pour la conduite des taillis à courtes rotations. 
Nous avons comparé :

- d'une part les accroissements ligneux au cours des premières années à différentes densités ;

- d'autre part, la production totale :

a) de 5 rotations de 2 ans et du témoin à 10 ans,

b) de 3 rotations de 3 ans et du témoin à 9 ans,

c) de 2 rotations de 4 ans et du témoin à 8 ans.

Notons que les accroissements courants ont été acquis en calculant la différence des volumes sur pied entre 2 relevés, et en divisant cette différence par le nombre d'années écoulé entre ces mesures.

\section{Résultats}

\subsection{Expérimentation $n^{\prime \prime} 1$ (fig. $1,2,3,4$ )}

Pour un écartement de $3,5 \times 3,5 \mathrm{~m}$, la production ligneuse en Mamora Centrale n'excède pas $100 \mathrm{~m}^{3} /$ ha au cours des 12 premières années. Le témoin $\mathrm{T}$ atteint $94 \mathrm{~m}^{3} / \mathrm{ha}$, tandis que le traitement T 2 n'atteint que $62,3 \mathrm{~m}^{3} / \mathrm{ha}$, soit une production plus faible de 34 p. 100 (fig. 1). En revanche, T 4, T 6, T 8 ont des productions totales ligneuses qui dépassent largement, mais non significativement $(P=0,05)$ $\mathrm{T}$ à 12 ans.

La figure 2 montre que l'accroissement moyen annuel semble plafonner au mieux, après l'âge de 4 ans aux alentours de $8 \mathrm{~m}$ '/ha/an. Mais, lorsque l'exploitation a lieu tous les 2 ans, celui-plafonne à moins de $6 \mathrm{~m}^{3} / \mathrm{ha} / \mathrm{an}$. L'accroissement moyen des arbres exploités tous les 4 ans (T 4) diffère peu des témoins, mais leur accroissement courant à 8 ans, c'est-à-dire en 1976 (après la sécheresse ?) est supérieur.

Sur la figure 3, il apparaît encore plus nettement qu'au cours des 2 années qui suivent la coupe rase d'un peuplement, l'accroissement moyen ligneux des rejets est inférieur à celui des témoins âgés de 4 ans ou plus. Au cours des 10 premières années, $T 10$ et $T$ présentent des accroissements très voisins. Mais, au cours des 2 années qui suivent la coupe à blanc de $\mathrm{T} 10$, l'accroissement ligneux de ce traitement n'est que de $12,5 \mathrm{~m}^{3} / \mathrm{ha} /$ an contre $20,2 \mathrm{~m}^{3} /$ ha pour le témoin $\mathrm{T}$.

En revanche, on constate souvent que, 4 ans après le recépage, l'accroissement moyen des peuplements recépés est supérieur à celui du témoin $\mathrm{T}$. Tel est le cas pour les 2 dernières rotations de $\mathrm{T} 4$, la dernière rotation de $\mathrm{T} 6$ et de $\mathrm{T} 8$.

Deux ans après le premier recépage, la circonférence moyenne des brins est sensiblement la même, que ce premier recépage intervienne pour un système racinaire âgé de 2 ans, 4 ans ou 10 ans (fig. 4).

Notons enfin qu'à 10 ans, la biomasse aérienne totale sèche de $T$ atteint $60 \mathrm{t} / \mathrm{ha}$ dont 2,2 $\mathrm{t}$ pour les fruits et les inflorescences, $2,6 \mathrm{t}$ pour les feuilles et 55,2 $\mathrm{t}$ pour la partie ligneuse. 
Volume Ligneux
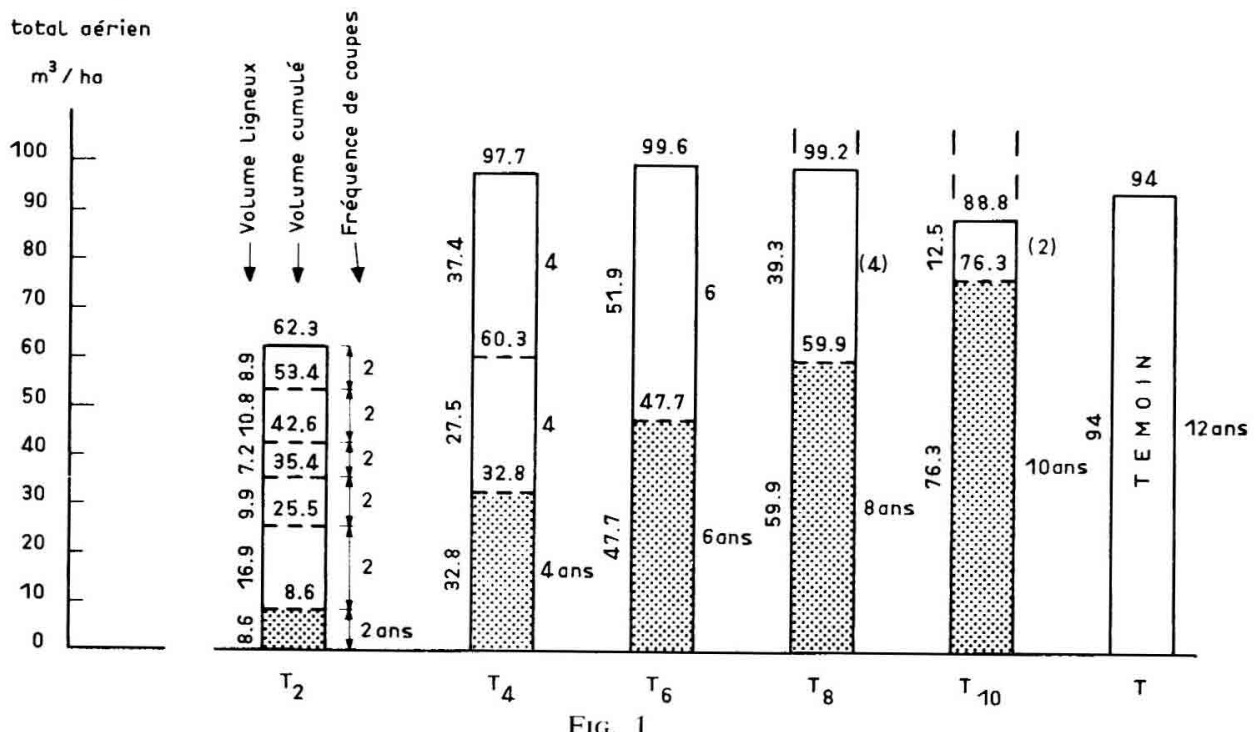

Influence de la fréquence de coupe sur le volume ligneux aérien total produit en 12 ans ( $T=$ témoin ; T 2, T 4, T 6, T \&, T $10=$ pcuplements coupés tous les 2, 4, 6, 8 ou 10 ans).

Effect of rotation length on total above ground wood production at 12 years ( $T=$ control ; T 2, T 4, T 6, T 8, T 10 stands cut every $2,4,6,8$ or 10 years).
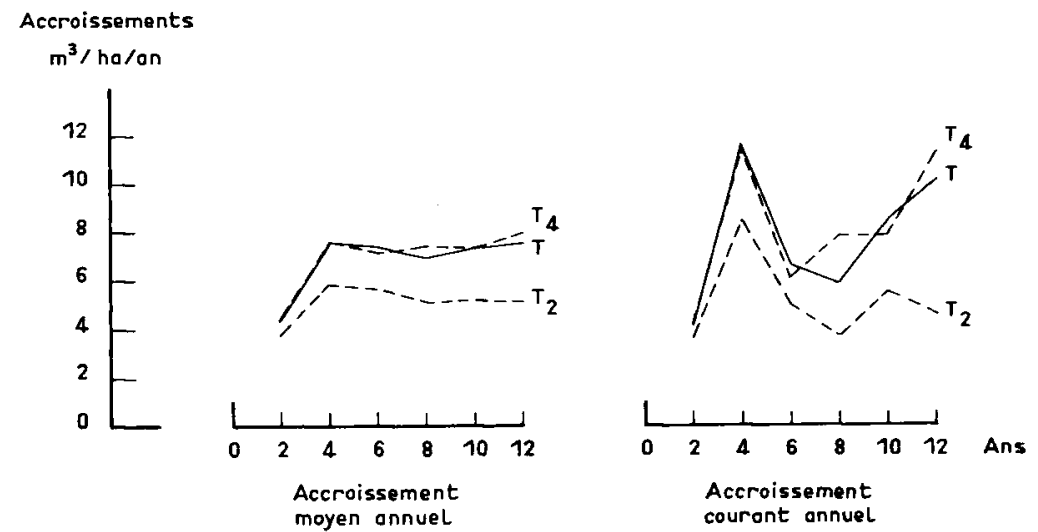

FIG. 2

Accroissement moyen ammel et accroissement courant annuel on volume ligneux airien selon la fréquence d'exploitation

$(T=$ témoin; $T 2$ et $T 4$ partie de peuplement exploitée tous les 2 ou 4 ans).

Variation of mean annual increment and carrent annual increment of above ground wood according to rotation length $(T=$ control ; $T 2-T 4$ stands cut every 2 or 4 years $)$. 


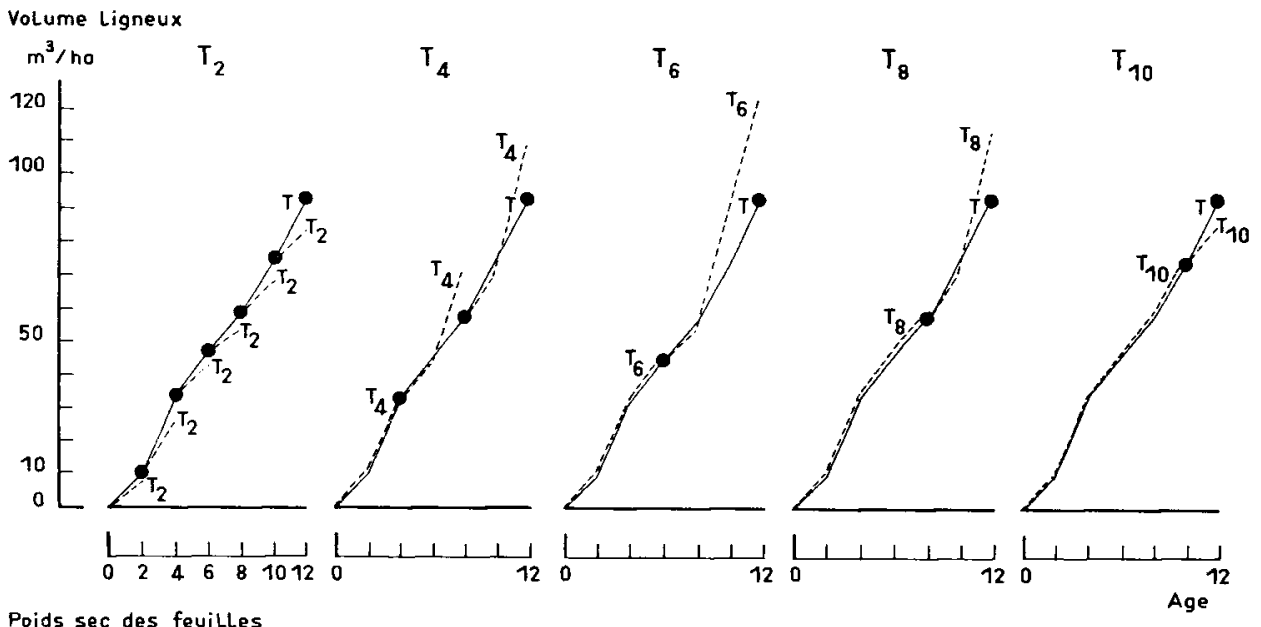

Poids sec des feuilles

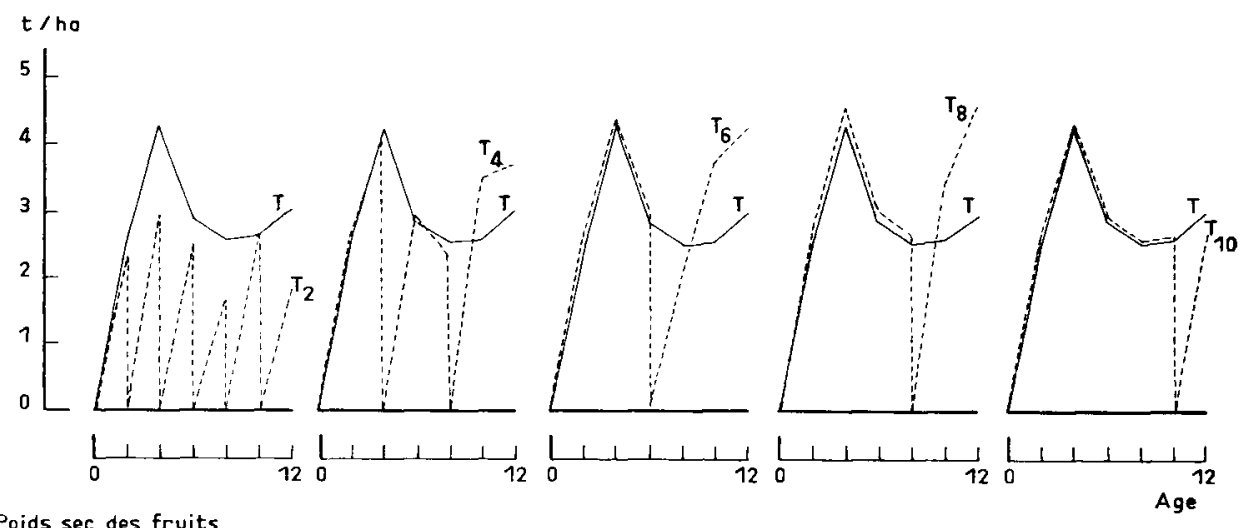

Poids sec des fruits

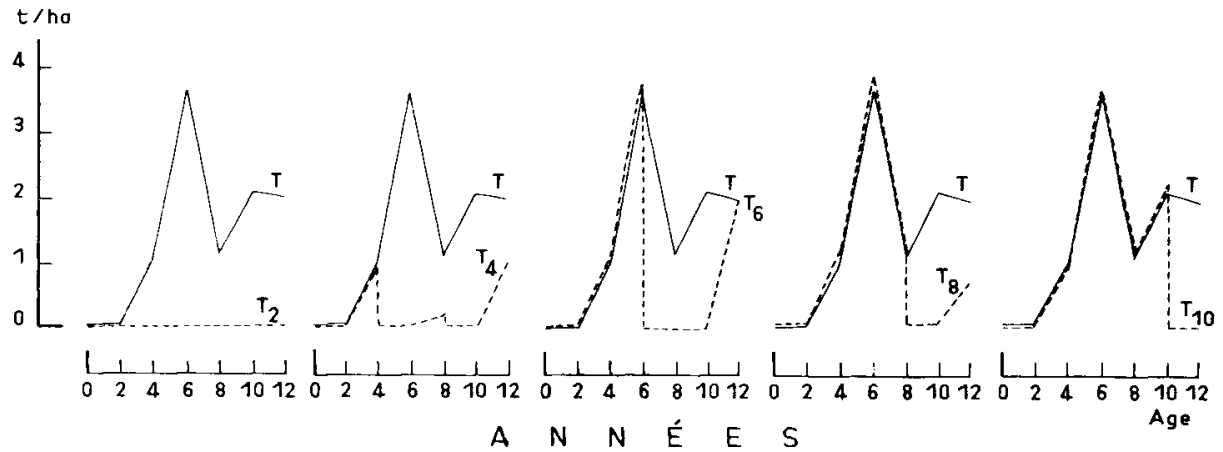

FIci. 3

Acrion de la fréquence de coupe sur :

Effect of rotation length on :

(En haut) : l'évolution du volume ligneux (en trait plein : accroissements cumulés en volume témoin - en pointillé : accroisscments cumulés des taillis coupés tous les 2 ans T 2 ; tous les 4 ans T 4, tous les 6 ans T 6 , ou tous les 10 ans T 10 ; pour chaque nouvelle rotation on a pris comme point de rélérence le volume de la futaie au moment de la coupe.

(Au milieu) : l'évolution du poids sec des feuilles.

(En bas) : l'évolution du poids sec des fructifications.

(Top) above ground wool volume (full line $=$ volume of the control - dotted lines : cumulated wood increments for coppiced stands cut every 2 years $(T 2)$, every 4 vears $(T 4)$. every 6 years (T 6), every 8 years $(T 8)$ and every 10 years $(T 10)$; for each new rotation we took the volume of the control as a new origin).

(Middle) : variation of leaf dry weigth.

(Bottom) : variation of fructification dry weigth. 


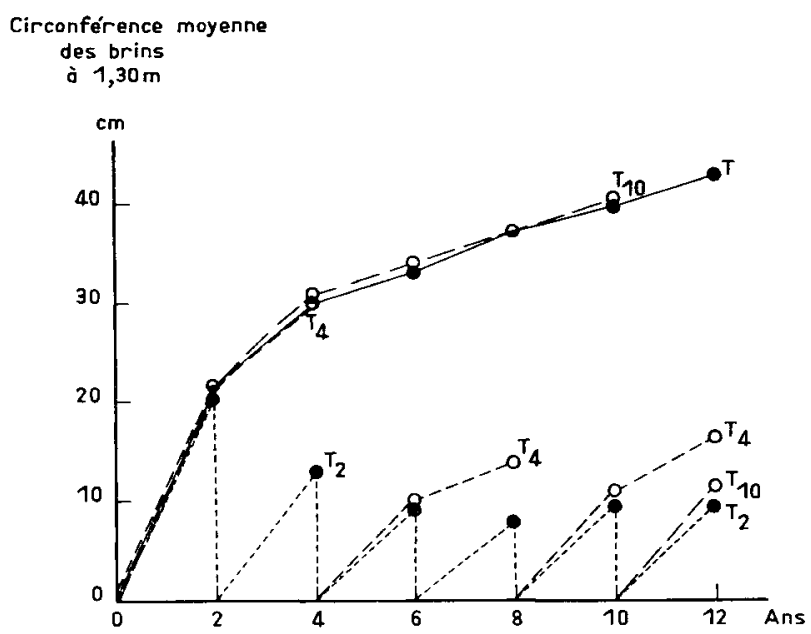

FIG. 4

Evolution de la circonférence moyenne des tiges suivant le traitement ( $T=$ témoin ; T 2, T 4, T 10 peuplements coupés tous les 2, 4 ou 10 ans).

Evolution of mean girth of shoots at breast heigth according to the treatment ( $T=$ control; T 2, T 4, T 10 stands cut every 2,4 or 10 years).

Pour la masse foliaire, on note dans tous les cas l'existence d'un maximum à l'âge de 4 ans (fig. 3). Chez les témoins, ce maximum dépasse $4 \mathrm{t}$ de M.S./ha. Mais, il chute ensuite jusqu'à 3 t/ha. Et, tandis que cette masse foliaire diminue, la quantité de fruits produite - qui était négligeable au cours des deux premières années suivant la plantation - augmente et atteint son maximum (plus de 3,5 t) à 6 ans (fig. 3).

Lorsque le recépage a lieu tous les 2 ans (T 2), la masse foliaire n'atteint toujours que des valeurs inférieures au maximum possible sur la station pour cette densité de plantation ( 2 à $3 \mathrm{t} / \mathrm{ha}$ au lieu de $4 \mathrm{t} / \mathrm{ha}$ ), tandis que la production de fruits reste pratiquement nulle.

En revanche, lorsque le recépage a lieu tous les 4 ans (T 4), ou 6 ans (T 6), ou 8 ans (T 8), la masse foliaire, 4 ans après, est égale au maximum antérieur constaté dans les témoins $(T)$ et supérieure à l'état actuel de ces témoins. Mais, il y a très peu de fructifications 4 ans après la coupe (fig. 3).

Notons aussi que la perte d'ensouchement (exprimée en p. 100 du nombre de souches initial) au cours des rotations successives est faible $(1$ p. 100 pour $T$, 3,4 p. 100 pour T 10 et T $8,1,3$ p. 100 pour T $6,2,6$ p. 100 pour T 4 et 4,1 p. 100 pour T 2).

\subsection{Expérimentation $n^{\prime \prime} 2$ (fig. $5,6,7,8$ )}

La figure 5 montre qu'aussi bien à 8, 9, 10 ans, la production ligneuse maximale est atteinte pour les peuplements témoins dont la densité est supérieure ou égale à 
$10000 \mathrm{plants} /$ ha (écartement $1 \times 1 \mathrm{~m}$ ). Dans la même station, la production atteint $10 \mathrm{~m}^{3} / \mathrm{ha} /$ an d'accroissement moyen pour les plantations les plus denses contre seulement $4 \mathrm{~m}^{3} / \mathrm{ha} /$ an pour les écartements de $3 \times 3 \mathrm{~m}$ (1111 plants/ha).

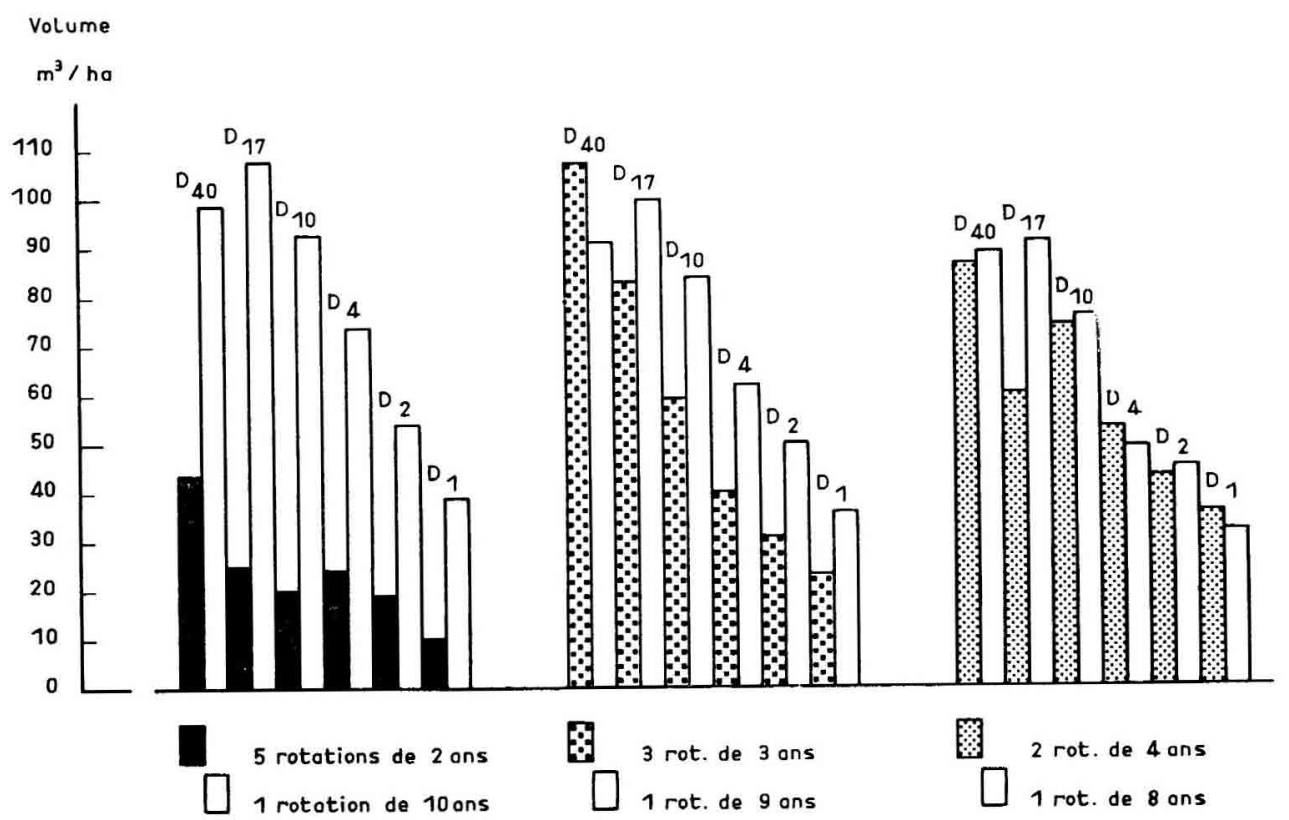

FIG. 5

Production comparée des témoins et des taillis coupés tous les 2 ans (à gauche), tous les 3 ans (au centre) ou tous les 4 ans (à droite) pour des densités variables $(D 1=1111$ plants/ha $(3 \times 3 \mathrm{~m}) ; D 2=2.220$ plants/ha $(1,5 \times 3 \mathrm{~m})$; D $4=4440$ plantsiha $(1,5 \times 1,5 \mathrm{~m}) ; D 10=10000$ plants $/ \mathrm{ha}(1 \times 1 \mathrm{~m})$; $D 17=17770$ plants $/ \mathrm{ha}(0,75 \times 0,75 \mathrm{~m}) ; D 40=40000$ plants/ha $(0,5 \times 10,5 \mathrm{~m})$.

Production of controls at 10,9 and 8 years compared to production of stands coppiced every 2 years (five times) on the left, every 3 years (three times) in the middle and every 4 years (two times) on the right $(D I=1111$ trees/ha: $D) 2=2220$; $D 4=4440 ; D 10=10000 ; D 17=17770 ; D 40=40000)$.

Lorsqu'on exploite ces taillis tous les 2 ans (fig. 5 à gauche), on constate, quelle que soit la densité de plantation, une chute de production par rapport au témoin supérieur à 60 p. 100. Par contre, lorsque les peuplements sont exploités tous les 4 ans (fig. 5 à droite), leur production totale ne diffère pas sensiblement des témoins. La densité de plantation à 17777 plants/ha fait exception, mais faute de répétitions, nous considérons que ce résultat est sans doute sans signification.

Lorsqu'on coupe les peuplements tous les 3 ans (fig. 5 au centre), la situation est intermédiaire entre celles qui viennent d'être décrites. La production totale est 
légèrement inférieure à celle des témoins, sauf peut-être pour la densité de plantation la plus élevée (40000 plants/ha), où elle est voisine du témoin.

La figure 6 montre que l'accroissement courant maximum en volume de la partie ligneuse aérienne atteint plus de $12 \mathrm{~m}^{3} / \mathrm{ha} / \mathrm{an}$ aux alentours de 3 ans et en tous les cas avant l'âge de 6 ans pour les plus fortes densités (40000 plants/ha et 17777 plants/ha). Dans les densités plus faibles, le maximum est atteint après 8 ans. A l'âge de 10 ans, l'accroissement courant de la densité la plus faible (1 111 plants/ha) n'atteint encore que $4 \mathrm{~m}^{3} / \mathrm{ha} / \mathrm{an}$, tandis que celui des plantations les plus denses avoisine $10 \mathrm{~m}^{3} / \mathrm{ha} / \mathrm{an}$.

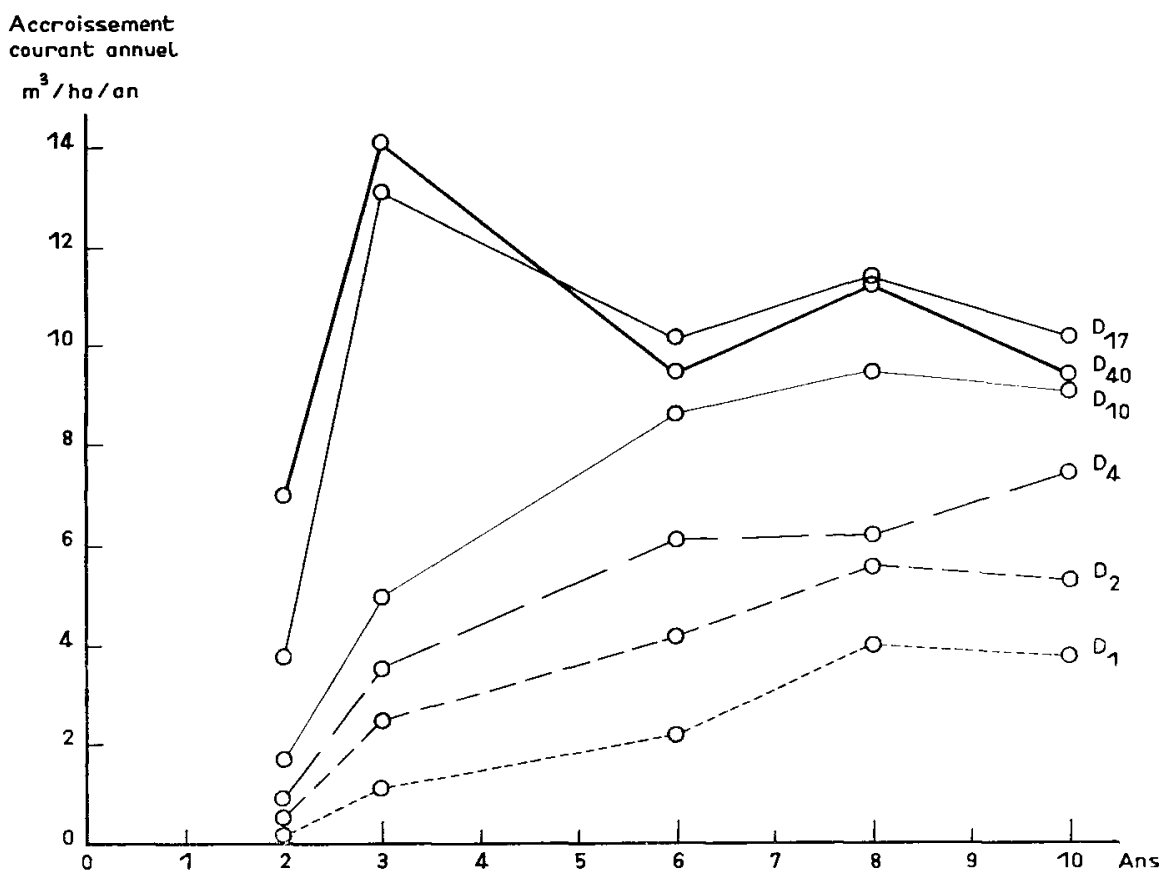

FIG. 6

Evolution de lacroissement courant annuel du volume ligneux aérion de futaies pour différentes densités (D 1,D2,D 4,D 10,D 17, D 40 voir légende fig. 5).

Variation of current annual increment of above ground wood of hight forests with different spacings (D 1,D 2,D 4, D 10,D 17, D 40 see fig. 5).

Comme dans les expériences précédentes, la masse foliaire atteint son maximum relativement tôt, entre 3 et 6 ans, sauf pour l'écartement $3 \times 3 \mathrm{~m}$ (fig. 7). Dans ce dernier cas, elle augmente toujours, et lentement, jusqu'à l'âge de 10 ans. A faible écartement $(0,5 \times 0,5 \mathrm{~m}, \mathrm{D} 40)$ elle atteint plus de $5 \mathrm{t} /$ ha dès l'âge de 3 ans. On 
remarquera qu'à cet âge, elle dépend fortement de la densité de plantation ; on trouve respectivement $5,9 \mathrm{t} ; 5,3 \mathrm{t} ; 5,2 \mathrm{t} ; 3,1 \mathrm{t} ; 2,1 \mathrm{t}$; et $0,8 \mathrm{t}$ de feuilles/ha pour les écartements $0,5 \times 0,5 \mathrm{~m} ; 0,75 \mathrm{~m} \times 0,75 \mathrm{~m} ; 1 \times 1 \mathrm{~m} ; 1,5 \times 1,5 \mathrm{~m} ; 1,5 \times 3 \mathrm{~m}$; et $3 \times 3 \mathrm{~m}$.

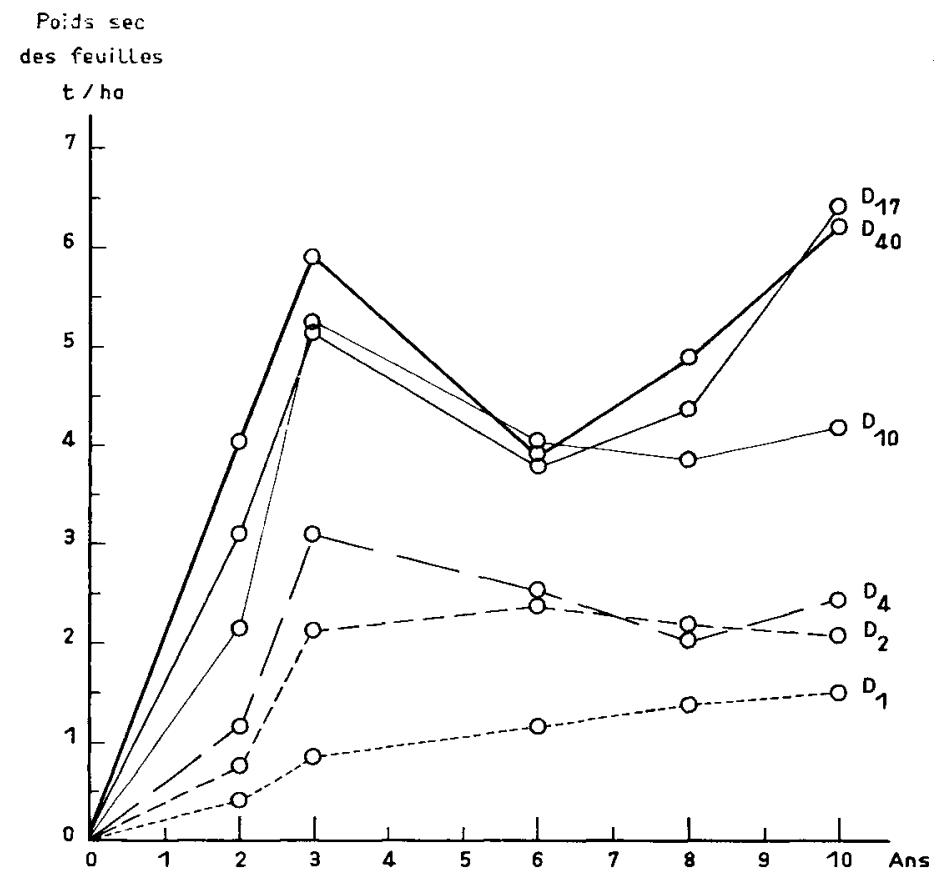

FIG. 7

Evolution du poids sec des feuilles par hectare de futaies plantées à différentes densités (D) $1, D 2, D 4, D 10, D 17, D 40$ voir fig. 5).

Variation of leaf dry matter/ha of high forests with different spacing (D 1, D 2, D 4,D 10,D 17, D 40 se fig. 5).

A 6 ans, après la sécheresse de 1976, les valeurs se regroupent respectivement vers $4 \mathrm{t} /$ ha pour les écartements les plus faibles $(0,5 \times 0,5 \mathrm{~m} ; 0,75 \times 0,75 \mathrm{~m}$; $1 \times 1 \mathrm{~m})$, vers $2,5 \mathrm{t} /$ ha pour les écartements intermédiaires $(1,4 \times 1,5 \mathrm{~m}$ et $1,5 \times 3 \mathrm{~m})$.

Pour l'écartement le plus grand $(3 \times 3 \mathrm{~m})$, il n'y a à 6 ans et ensuite qu'un peu plus d'une tonne de feuilles par hectare. Par la suite, la masse foliaire des écartements les plus faibles $(0,5 \times 0,5 \mathrm{~m} ; 0,75 \times 0,75 \mathrm{~m})$ augmente de nouveau.

Lorsque les pcuplements sont coupés tous les 2, 3 ou 4 ans, la production foliaire la plus importante est obtenue pour les densités de plantation les plus fortes (écartements : $0,5 \times 0,5 \mathrm{~m}$ ) (fig. 8). Les maximas sont atteints pour des rotations de 3 ans. 


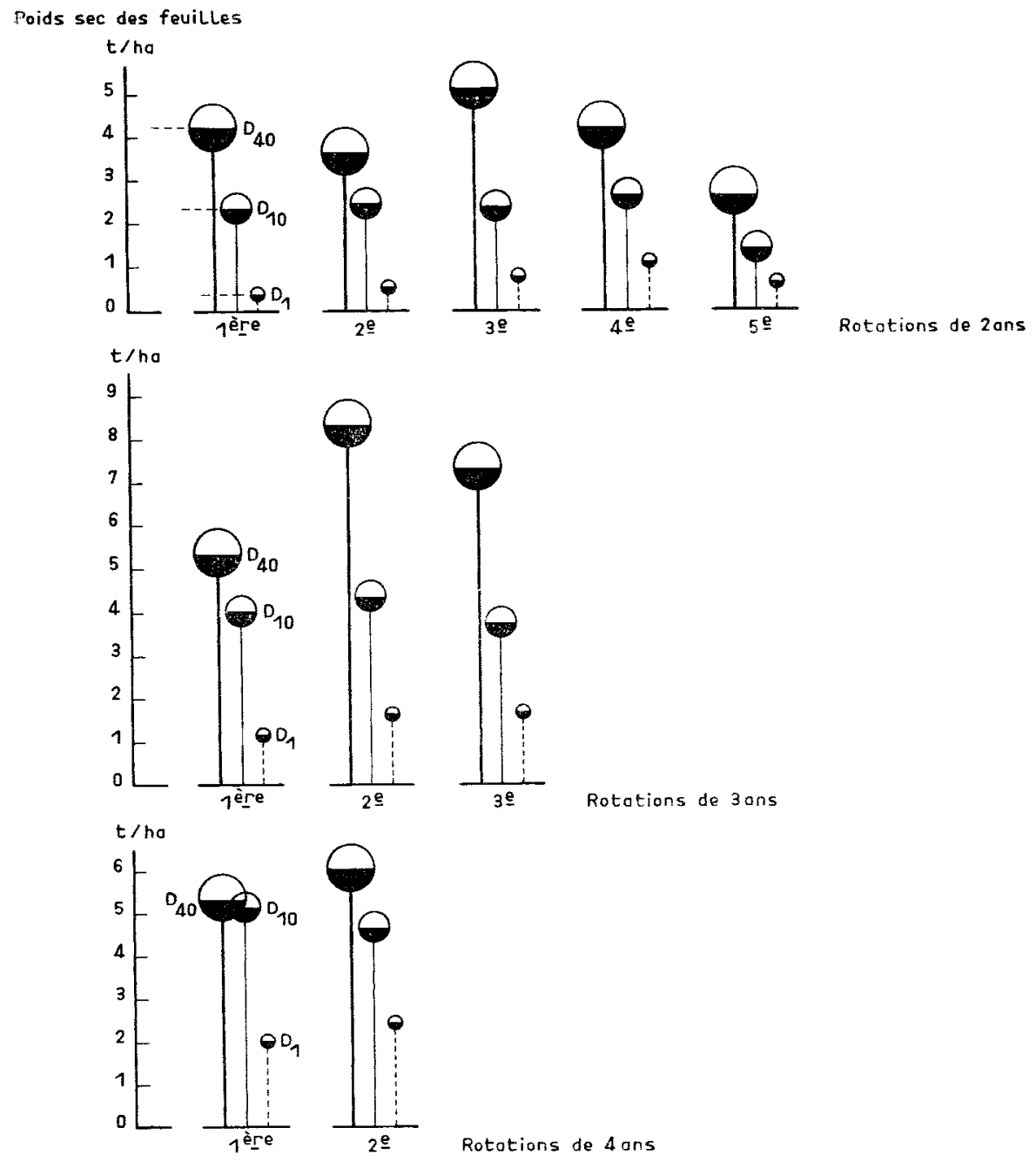

FIG. 8

Poids sec des feuilles en t/ha à la fin de différentes rotations pour trois densités de plantation $(D 1=1111$ plants $/$ ha $; D 10=10000$ plants $/$ ha $; 040=40000$ plants $/$ ha $)$.

Leaf dry weigth in $t / \mathrm{ha}\left(10^{3} \mathrm{~kg} / \mathrm{ha}\right)$ at the end of different rotations $(t o p=2$ year rotations - Middle $: 3$ year rotations - Bottom : 4 year rotations $)$ for three tree densities $(D)=1111$ trees $/$ ha $; D 10=10000$ trees $/$ ha and $D 40=40000$ trees/ha $)$. 


\section{Discussion}

\subsection{Effet de la densité de plantation sur la production ligneuse aérienne}

Les résultats présentés ci-dessus montrent qu'en forêt de la Mamora, sous climat semi-aride et sur un sol peu fertile, dans le cadre observé - 0 à 12 ans on peut augmenter la production ligneuse des peuplements en augmentant la densité de plantation. Un écartement de $1 \times 1 \mathrm{~m}$, soit 10000 plants à l'ha, est sans doute proche de la densité de plantation optimum. Lorsque la densité de plantation est plus faible, la production ligneuse est aussi plus faible. En revanche, lorsque la densité de plantation est plus élevée, l'accroissement de production n'est plus significatif.

\subsection{Effet de la fréquence de coupe sur la production ligneuse aérienne}

Mais, quelle que soit la densité de plantation, il apparaît aussi qu'on n'a pas intérêt à exploiter le taillis trop fréquemment. Dans les stations étudiées, l'exploitation peut être faite tous les 4 ans sans perte de production ligneuse. Une exploitation plus fréquente - tous les 3 ans ou, pire, tous les 2 ans - entraine une baisse de production pouvant aller jusqu'à 60 p. 100 par rapport au témoin.

Ces résultats confirment également ceux que STEıNBECK et BRown (1976) avaient trouvés pour les premières exploitations de taillis de platanes aux Elats-Unis.

Lorsque la coupe a lieu tous les 2 ans, l'appareil foliaire n'a pas le temps de se reconstituer intégralement ; cela pourrait être à l'origine de la baisse de production constatée. Lorsque l'exploitation d'un taillis planté à fort écartement a lieu tous les 4 ans, on obtient parfois un gain de production de bois. Cela pourrait être dû soit au fait que le recépage supprime alors, ou diminue fortement, la production de fructifications, soit au fait qu'il permet d'obtenir une masse foliaire plus importante que chez les lémoins. Mais, peut-être les deux phénomènes, production de fruits et production de feuilles, ne sont-ils pas indépendants?

Par ailleurs, nous avions précédemment constaté (RILDACKLR, 1973) que la production des taillis subissant des rotations de 10 à 14 ans baissait au cours de la troisième rotation, c'est-à-dire lorsque l'âge des souches atteignait une trentaine d'années. Cette baisse pouvait, selon nous, être due soit au recépage, soit au vieillissement des souches, soit à l'épuisement minéral de la station. Les expériences ci-dessus tendraient à prouver que la baisse de production serait plutôt déterminée par le vieillissement des souches. En effet, pour les rotations de 4 ans, on ne constate pas de baisse de production au cours de la troisième rotation (âge des souches : 12 ans en fin de troisième rotation). Et la production de notre taillis coupé tous les 2 ans, bien qu'inférieure à celle du témoin, ne diminue pas au fur et à mesure qu'augmente le nombre de recépage. En revanche, ces essais ne permettent pas encore de savoir si un recépage effectué par exemple tous les 4 ans ralentit ou accélère la senescence des systèmes racinaires par rapport à un recépage effectué par exemple tous les 15 ans.

\subsection{Variation de la masse foliaire et fertilité}

Les expérimentations décrites ont été réalisées sur 2 stations dont la fertilité diffère. Sur sol fertile (sable rouge - expérimentation 1), à écartement $3,5 \times 3,5 \mathrm{~m}$, 
la masse foliaire culmine vers l'âge de 4 ans, atteignant entre 3 et 4 t/ha. Sur sol peu fertile (sable beige, expérimentation 2), à écartement voisin, la masse foliaire $(3 \times 3 \mathrm{~m})$ est plus faible $(1,5 \mathrm{l} / \mathrm{ha})$ et semble augmenter encore à l'âge de 10 ans. Mais, cette masse foliaire est beaucoup plus forte (plus de $5 \mathrm{t} / \mathrm{ha}$ ) dans la même station pour des écartements plus faibles $(0,5 \times 0,5 \mathrm{~m})$. Ainsi, il semblerait que l'on puisse nettement accroitre la production sur une station peu fertile en augmentant la densité de plantation. Il est probable que cela amćliore la prospection du sol par les racines des arbres et augmente de ce fait l'indice foliaire.

Le niveau de l'indice foliaire pourrait done dépendre non seulement de la fertilité de la station (Decourt, 1973), mais encore de la densité de plantation. Les études concernant les taillis devraient, pensons-nous, préciser le niveau et les variations de masses foliaires suivant les traitements, afin de pouvoir ultérieurement micux comprendre quels sont les facteurs responsables de la productivité des peuplements.

\subsection{Conséquences pratiques}

En Mamora Centrale, la production ligneuse aérienne maximale peut être atteinte pour des densités de plantation voisines de 1000 plants/ha $(1 \times 1 \mathrm{~m})$, et cela que le peuplement soit traité en futaie ou en taillis, à condition que dans ce dernier cas les rotations ne soient pas inférieures à 4 ans.

Le traitement en taillis donne des produits de plus petits diamètres, donc plus facilement récoltables mécaniquement. Mais, cette production comprend aussi davantage d'écorce, ce qui peut être un inconvénient lorsque le bois doit être écorcé, notamment pour la production de certaines qualités de pâte à papier.

\section{Summary}

Wood production of Eucalyptus coppiced stands : effect of rotation length and spacing

Total above ground wood production of Eucalyptus camaldulensis in Morocco is influenced by spacing and rotation length.

In a latin square, at 12 years, for a $3,5 \times 3,5 \mathrm{~m}$ spacing, total production of controls (non coppiced) is not significantly different from squares cul every 4, 6, 8 or 10 years. But total production decreased $34 \mathrm{p} .100$ in squares cut every 2 years.

In another 10 year old trial, total wood production of non coppiced squares increased from $40 \mathrm{~m}^{n} /$ ha to $100 \mathrm{~m}^{: / h}$ ha when tree density increased from $1 / 11 / \mathrm{ha}$ up to $10000 / \mathrm{ha}$. But this production no longer increased with higher tree densities ranging from $10000 /$ ha to $40000 /$ ha. As in the first experimental plot, coppicing did decrease total production only when rotation length was shorter than 4 years and this for all spacings. Total wood production of five 2 year rotations ranges from $25 \mathrm{p}$. 100 to $40 \mathrm{p} .100$ of the production obtained with the other treatments.

Leaf weight evolution and fruit weight evolution partly explain these results. Origin of decrease of wond production after three 10 year or more rotations is discussed. 


\section{Références bibliographiques}

Artigues R., Lipoufre B., 1968. Contribution à la connaissance de l'Ecologic d'Eucalyptas camaldulensis dans les sols sableux de la Mamora. Ann. Rech. For. Maroc, t. 11, 299-327.

Bouchon J., RLidncker A., 1973. Allometric relationship in a young coppice of Eucalyphus camaldulensis. Indications for the above ground biomass sampling. Biomass studics. I.U.F.R.O. S4.01 et S4.02 - Symposium Nancy - Vancouver 1973, H.E. Young, Editor University of Maine, p. 259-267.

Decourl N., 1973. Production primaire, production utile : méthode d'évaluation, indices de productivité. Ann. Sci. For., 30 (3), $219-238$.

RIEDACKi:R A., 1972. Physiologie des souches d'Eucalyptus comaldulensis traités en taillis au Maroc. Le problème de leur vieillissement. Comptes rendus du $7^{*}$ Congrès Forestier Mondial, Bnenos-Aires, 4-18 octobre 1982, 1868-1874.

Ruedncker A., 1973. Les Lailis d'Eucalyptus au Maroc. Ann. Rech. For. Maroc, tome 13, 1973 , p. $157-349$.

STEINBECK K., BROWN C.L., 1976. Yicld and utilization of hardwood fiber grown on short rotations. School of Forest Resources, The University of Georgia, Athens, Georgia 30602. 